\title{
Liquid rotary jet fragmentation of a pressure atomizer
}

\author{
Alix Bartoli*1,2,3 Séverine Tomas $^{1}$, Bruno Molle ${ }^{1}$, Muriel Amielh ${ }^{2}$, Catherine Gibert ${ }^{3}$ and \\ Fabien Anselmet ${ }^{2}$ \\ ${ }^{1}$ INRAE, UMR G-EAU, Montpellier, France \\ ${ }^{2}$ Aix-Marseille Université, CNRS, Centrale Marseille, IRPHE UMR 7342, Marseille, France \\ ${ }^{3}$ Rolland arroseurs sprinklers, Mognard, France \\ ${ }^{*}$ Corresponding author email: alix.bartoli@inrae.fr
}

\begin{abstract}
Rotary water jets are commonly used in the world of agriculture, particularly in irrigation and in treatment with pesticides in the form of micro-sprinklers. However, their study is not very well developed, even though the problems linked to water resource losses or the drift of pesticides in the atmosphere are well known. Controlling the size distribution of the drops that are generated becomes essential to avoid these problems. The observation of the fragmentation of a rotating jet is implemented in this study. The shadowgraphy method coupled with a drop size and velocity detection algorithm has been adapted for this rotating system. Different modes of rupture can be observed and drop size distributions are analyzed. Primary atomization is controlled by the orthoradial air velocity generated by the rotation and by the stretching of the ligaments due to the Coriolis and centrifugal forces. We compare the different modes of fragmentation as a function of the aerodynamic forces created by the rotation and the momentum flux of the water jet in the air.
\end{abstract}

\section{Keywords}

Rotary jet, Primary atomization, Bag breakup

\section{Introduction}

Atomization of a liquid jet is encountered in many natural, industrial and agricultural phenomena [1],[2],[9]. Furthermore, in general, climate change requires an increasingly virtuous use of water resources. However, current spraying techniques for both pesticides and irrigation water are not yet optimal because they induce two phenomena: loss through drift into the atmosphere and run-off. In addition, the use of pesticides induces environmental and health risks related to drift. These issues therefore require the development of precision agriculture both in the field of irrigation and in the field of crop treatment. In these two fields, the optimization of sprinkling methods is partly based on the control of the dispersion of drops in the air. Droplets smaller than $100 \mu \mathrm{m}$ are sensitive to air drift while drops larger than $2.5 \mathrm{~mm}$ are likely, due to kinetic energy, to erode soils. Our study focuses on rotary irrigation sprinklers with the objective of managing the range of drops produced to avoid both drift and runoff. These sprinklers, called microsprinklers, are used at low pressure (1 to 2 bars). The rotation of the micro-sprinkler driven by the momentum flux at a specific pressure allows a very high water coverage in relation to the energy supplied. The other advantage is the generation of a more mono-disperse droplet size distribution than for the jets of other types of sprinklers. An experimental laboratory facility has been built to observe the fragmentation of a liquid jet with different angular velocities and nozzle sizes. We chose to control the angular velocity by using a motor and then to simplify the geometry by using a circular section nozzle. We implement a shadowgraphy method combined with DTV (Droplet Tracking Velocimetry) to observe the propagation, atomization and dispersion of the jets for this rotating system.

\section{Material and methods}

The design of the experimental bench focused on the possibility of performing shadowgraphy observations along the trajectory of the jet in a rotating frame of reference. The axis of rotation 
is the $z$ axis. The jet is channeled into the rotor so that the jet is emitted in the horizontal plane $(x, y)$. The jet is therefore rotating in the horizontal plane and subject to the gravity force. A $135 \mathrm{~mJ}(532 \mathrm{~nm}) \mathrm{Nd}-\mathrm{YAG}$ double pulse laser combined with a Dantec Dynamics ShadowStrobe laser diffuser is placed below the jet. The dual-frame PIV/DTV camera is placed above the plane described by the rotating jet. The complexity of observing a rotating jet is due to its movement in space as a function of time. The rotational movement of the jet curves the trajectories. This makes it difficult to observe it in a fixed frame. We have therefore placed a measuring device (Dantec cyclic synchroniser) which analyzes the rotation sensor signal (ifm RUP500) fixed to the rotation axis and has the capacity to detect the angle of rotation $\alpha$ of the nozzle in relation to a reference point, with an accuracy of $0.1^{\circ}$. We can then define the triggering of the camera/laser duo for a precise angle. Moreover, the camera and the laser are fixed on a 3D displacement bench $(x, y, z)$ with a precision of $6.25 \mu \mathrm{m}$. The displacement information $(x, y, z)$ relative to a reference point $\left(x_{0}, y_{0}, z_{0}\right)$ combined with the rotation angle $\alpha$ allows us to move along the trajectory of the jet (see Figures $1 \mathrm{a}$ and $1 \mathrm{~b}$ ). Depending on the rotation speed, we can define an acquisition every $n$ revolutions. The jet is turbulent at the nozzle exit, with a Reynolds number defined by $R_{e}=U_{b} d / \nu=O\left(10^{4}\right)$ where $d$ is the nozzle diameter. About geometry of the nozzle, the ratio of length to orifice diameter is in between $l / d=[8.7-14.5]$. The liquid ejection velocity is modified by changing the flow rate which is controlled by a solenoid valve. The exit velocity of the liquid jet is $U_{b}=Q_{b} / A_{b}$ where $Q_{b}$ is the flow rate and $A_{b}$ is the area occupied by the fluid at the nozzle. We made the assumption that the fluid completely occupies the nozzle circular section according to the observations of shadowgraphy at the nozzle exit. The interior of the nozzle is not currently visible. The orthoradial velocity is $U_{\theta}=R \Omega$, with $R$ the rotor radius $(R=0.03 \mathrm{~m})$ and $\Omega$ the angular velocity, $\rho_{G}$ and $\rho_{L}$ being the air and water densities respectively. The rotation of the nozzle is controlled by a motor connected to a frequency variator. The rotation speed varies in the range $\Omega=[52.3-303.7] \mathrm{rad} / \mathrm{s}$. The Rossby number $R_{o}=U_{b} / U_{\theta} \simeq 1$ symbolizes in our case the ratio between the jet ejection velocity and the orthoradial velocity generated by the rotation. The liquid Weber number is expressed as $W e_{l}=\rho_{L}\left(U_{b}^{2}+U_{\theta}^{2}\right) d / \sigma=\left[10^{3}-10^{4}\right]$.

(a)

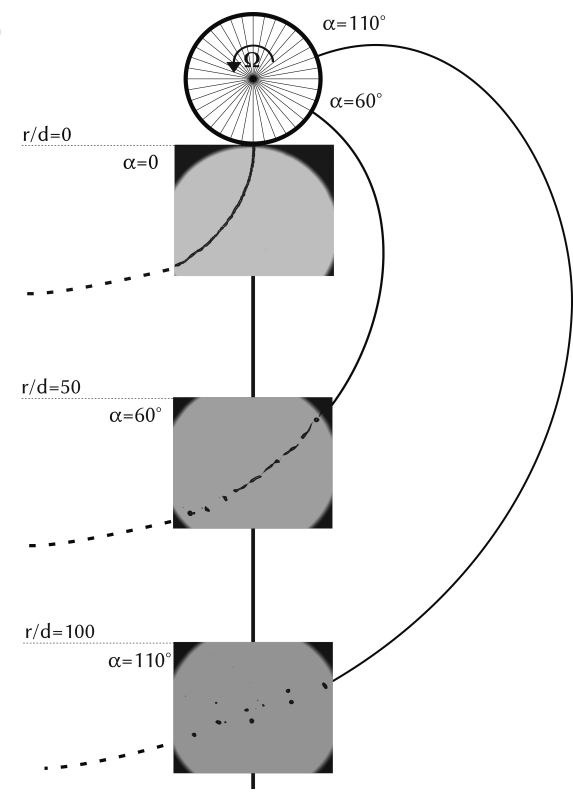

(b)

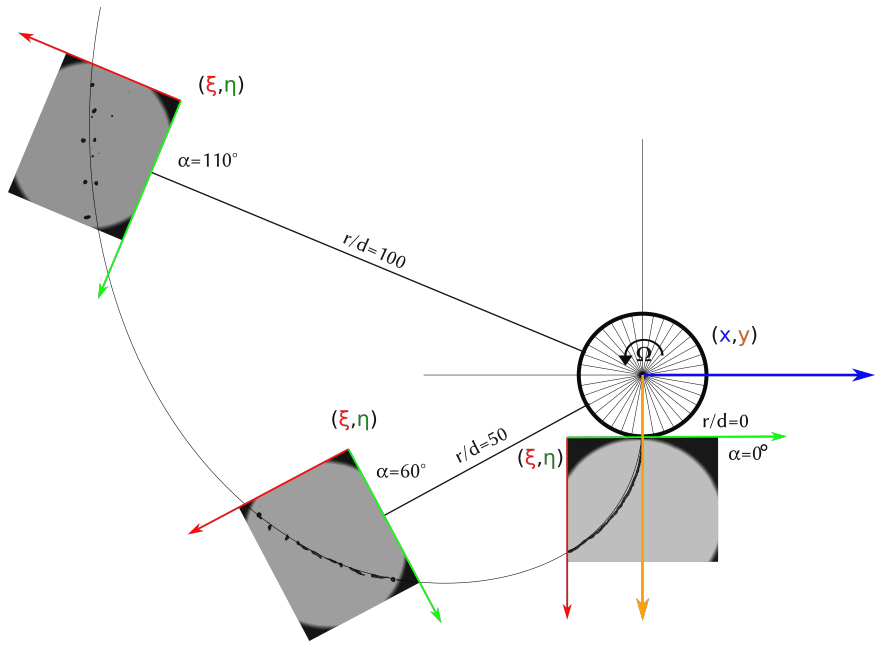

Figure 1. (a): The Figure illustrates the method used for observing the rotating jet. The angle $\alpha$ is defined by the cyclic synchronizer and the distance $r / d$ is adjusted by the displacement bench in order to observe each position of the jet. (b): The images captured from the local reference frame $(\xi, \eta)$ are then put back into the global reference frame $(x, y)$ of the laboratory to get the spiral trajectory of the rotating jet and the positions of the drops in this reference frame. 
The shadowgraphy method is used to observe the fragmentation of the jet. A 12-bit, 4M camera $\left(2048 \times 2048\right.$ pixels), with a Zeiss Milvus 2/100M lens, captures a field of view of $30 \times 30 \mathrm{~mm}^{2}$. The aperture is $\mathrm{f} / 2.0$ in order to obtain a depth of field less than $1 \mathrm{~mm}$. The spatial resolution is $14 \mu \mathrm{m} / \mathrm{pix}$ which allows the observation of drops with a minimum diameter of $50 \mu \mathrm{m}$. Shadowgraphy is combined with DTV (Droplet Tracking Velocimetry) to detect the sizes and velocities of water drops. The system captures two images with a time between pulses (tbp) in the order of $O(10 \mu \mathrm{s})$. The DTV is built using Dantec Dynamics' DynamicStudio software. A conventional method of detection and segmentation is used [3],[9]. For each acquisition, 1000 images are captured at a frequency $f=5 \mathrm{~Hz}$. A large population of drops is then detected (about $O\left(10^{4}\right)$ ). Several observation positions are defined with for each of them an angle of the cyclic synchronizer $\alpha$ and a displacement $\left(T_{x}, T_{y}\right)$ in order to be able to determine the trajectory of the jet, see Figure 1a. A change of reference frame is made from the local reference frame $(\xi, \eta)$ of the image, with the upper left corner of the image as the origin, to a global reference frame $(x, y)$ of the laboratory, with the centre of rotation as the origin. This translates and rotates the local coordinates, see Figure $1 \mathrm{~b}$ and equation (1). We then have :

$$
\left(\begin{array}{l}
x \\
y
\end{array}\right)=R_{\alpha}\left(\begin{array}{c}
\xi+T_{x} \\
\eta+T_{y}
\end{array}\right)
$$

with a rotation matrix $R_{\alpha}=\left(\begin{array}{cc}\cos (\alpha) & -\sin (\alpha) \\ \sin (\alpha) & \cos (\alpha)\end{array}\right)$, where $T_{x}$ and $T_{y}$ are the translations on the $x$ and $y$ axes respectively.

At each position, a check of the jet image sharpness is carried out because gravity bends the jet slightly according to the radius $r$, but this is very weak because the images are taken in a radius close to the centre of rotation (maximum $r=0.2 \mathrm{~m}$ ). The deformation of the jet by gravity is corrected with the help of the displacement bench on the $z$ axis (accuracy of $6.25 \mu \mathrm{m}$ ) so that the jet remains at the centre of the depth of field. To determine the trajectory, three different positions are chosen. The first position is at the nozzle to define the reference point $\left(x_{0}, y_{0}, z_{0}\right)$. The second position is determined visually at the breakup length and the third position is at the location of the fully atomized jet $(r / d=[125-150])$. The jet interface is captured using the same thresholding method as for drop detection. With the information from the jet interface and the mass centre of the drops, the central trajectory of the jet can be deduced. The parametric equation of the trajectory, see equation (2), is adjusted to the detected central trajectory using the three images taken at these different positions. The radial velocity and deceleration of the radial velocity of a particle in a rotating system are taken into account to compute the particle position $a(t)$, which is a second degree polynomial function of time.

$$
\left(\begin{array}{c}
x-x_{0} \\
y-y_{0}
\end{array}\right)=(R+a(t))\left(\begin{array}{c}
\cos \left(\Omega t_{0}+t\right) \\
\sin \left(\Omega t_{0}+t\right)
\end{array}\right)
$$

The breakup length is detected by the intersection between the interface of the last broken ligament and the central trajectory of the jet. The break point closest to the nozzle is the breakup point. 
The calculation of the breakup length is based on the curvilinear abscissa $s(t)=\sqrt{x(t)^{2}+y(t)^{2}}$ using the equation of the trajectory to the detected breakup point. The breakup length of the rotating jet can then be calculated as a function of the rotation speed and the exit velocity of the jet. The breakup radius is defined as the shortest distance between the edge of the rotor and the breakup point (see Figure $5 b$ which will be discussed in detail later on).

The observation and study of the velocity and size of the drops is carried out as a function of the angular velocity of the jet.

\section{Results and discussion}

Experiments were carried out with nozzle sizes $d=1.2,1.5$ and $2 \mathrm{~mm}$ and a flow rate varying between 35 and $120 \mathrm{~L} / \mathrm{h}$ to achieve all breakup modes. Rotation of the nozzle located at a radius $R$ causes a side wind at the level of the nozzle depending on the radius, $U_{\theta}=R \Omega$. Increasing the rotation speed increases the aerodynamic forces on the jet. At many points the rotating jet can be compared to a cross-flow jet. The same modes of fragmentation can be found in the articles on cross-flow jets [5],[8]. The aerodynamic Weber number $W e_{g}=\rho_{G} U_{\theta}^{2} d / \sigma$ and the momentum flux ratio $q=\rho_{L} U_{b}^{2} / \rho_{G} U_{\theta}^{2}$ describe the appearance of the different modes. Several breakup modes are observed in our study case: Rayleigh breakup, bag breakup and stretched breakup [7]. They can be grouped into three main categories knowing the difficulty of having a clear demarcation between these observations: Rayleigh breakup, multimode breakup (Rayleigh, bag and stretched breakup) and stretched breakup. Figure 2 shows these different modes of fragmentation. The difference between a cross-flow jet and our configuration is the addition of Coriolis and centrifugal forces which stretch the jet and therefore make it thinner. There is a balance between aerodynamic forces, surface tension and fictitious forces (Coriolis and centrifugal). At low aerodynamic Weber numbers, the jet is fragmented as in a Rayleigh regime. The ligament is stretched by Coriolis and centrifugal forces and the aerodynamic forces promote its rupture between the nodes of the ligament. When the aerodynamic force is high, the ligament will be in the form of a sheet. This will increase the drag, favouring its flattening and generating the bag breakup.

(a)

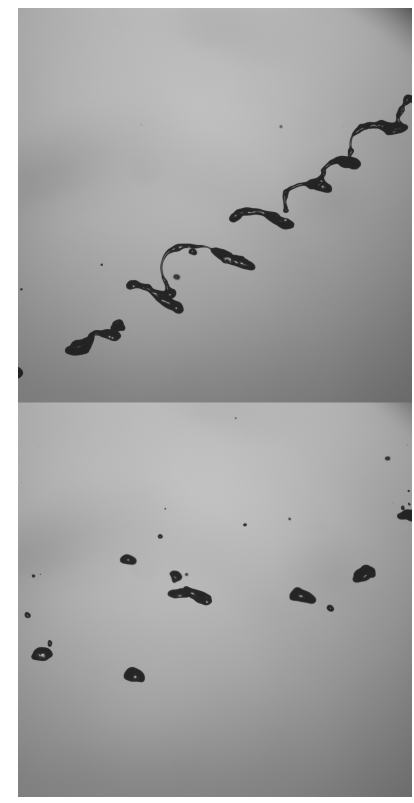

(b)

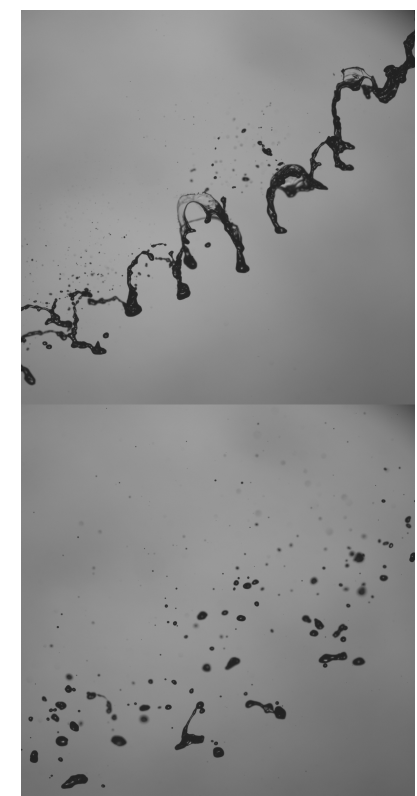

(c)

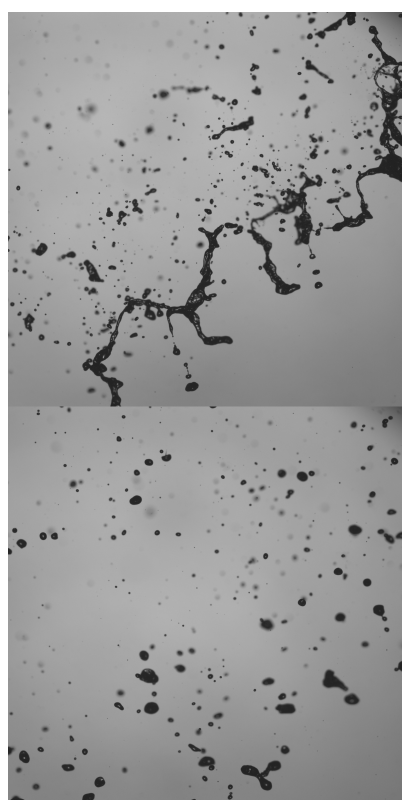

Figure 2. Different fragmentation modes for a rotating jet. One stands on the trajectory of the jet at an angle $\alpha$ and a displacement $r / d$. (a) Rayleigh breakup. This mode is obtained with $d=1.2 \mathrm{~mm}, Q=55 \mathrm{~L} / \mathrm{h}, \Omega=175 \mathrm{rad} / \mathrm{s}$ at $r / d=80$ and $\alpha=53^{\circ}$. The first image, at $r / d=125$ and $\alpha=97^{\circ}$, represents the fully fragmented jet. (b) Multimode breakup (bag and column breakup), $d=1.2 \mathrm{~mm}, Q=75 \mathrm{~L} / \mathrm{h}, \Omega=292 \mathrm{rad} / \mathrm{s}$ at $r / d=40$ and $\alpha=31^{\circ}$. The second image is at a distance of $r / d=125$ and $\alpha=138^{\circ}$. (c) Stretched breakup, $d=1.5 \mathrm{~mm}, Q=100 \mathrm{~L} / \mathrm{h}, \Omega=303 \mathrm{rad} / \mathrm{s}$ at $r / d=50$ and $\alpha=48^{\circ}$. The third image is at $r / d=133$ and $\alpha=98^{\circ}$. 
The ratio of the momentum fluxes influences the type of regime (see Figure 3). If $q$ is very large, one can consider the classical atomization regimes for a non-rotating jet. The effects of rotation are accentuated when $q \leq 2000$ and $W e_{g}>1$, and the bag breakup mode appears. When $q \geq 2000$ and $W e_{g}>2$, the stretched breakup mode is set up. We have the possibility to modify the flow rate, the nozzle size and the rotation speed to reach these different fragmentation modes. Figure 3 represents the different experiments carried out to study the rotating jet according to these different parameters. The particularity of the present study is the high momentum flux ratio $q=[500-9500]$. Therefore, even with relatively low aerodynamic Weber numbers, significant mode changes can occur.

The drop size distribution depends on the atomization mode. In the Rayleigh mode, the probability densities (pdf) of the drop sizes are bi-modal [4]. There is a strong distinction between satellite drops and main drops generated by the fragmentation of a ligament. In addition, when there are several combined modes of rupture, Rayleigh, bag and stretched breakup modes, the spread of the drop range is reduced and the peak is narrower. When the spray is fully fragmented at large $q$ and $W e_{g}$ in the stretched breakup mode, there is a single peak with high amplitude and a reduction in the drop size range.

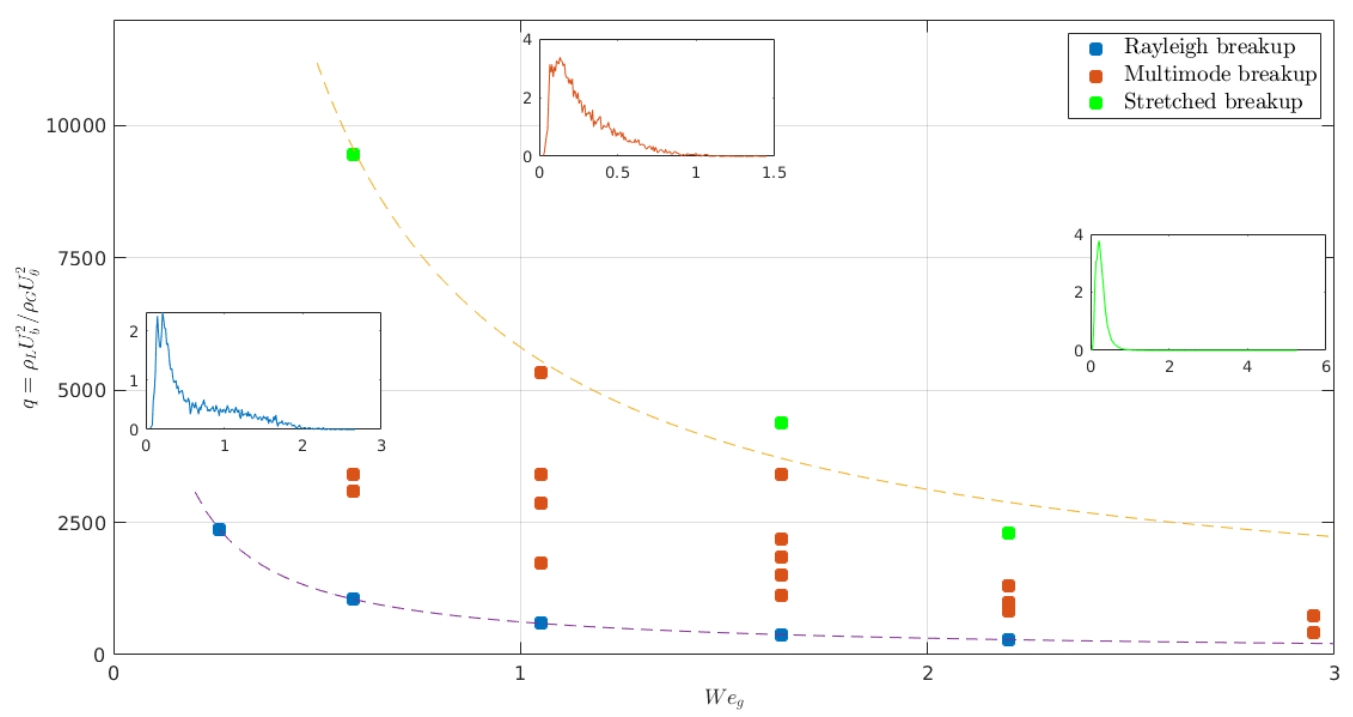

Figure 3. Results obtained for the different experiments carried out, according to the aerodynamic Weber number $W e_{g}$ and the momentum flux ratio $q$. The three modes of rupture are observed. For each mode, a typical drop size distribution $(\mathrm{mm})$ is represented. The dotted lines are a $1 / x$ curve and delimit the different regimes.

The characteristic diameters, the mean volume diameter $D_{30}=\left(\sum_{i=1}^{n} n_{i} d_{i}^{3} / \sum_{i=1}^{n} n_{i}\right)^{1 / 3}$ and the mean Sauter diameter $D_{32}=\sum_{i=1}^{n} n_{i} d_{i}^{3} / \sum_{i=1}^{n} n_{i} d_{i}^{2}$, are used to describe the jets under different conditions at the position where the jet is fully atomized $(r / d=[125-150])$ (see Figures $4 a$ and 4b). There is a decrease in the values of $D_{30}$ and $D_{32}$ depending on the rotation speed. The ligaments being stretched by centrifugal and Coriolis forces become thinner and generate smaller drops in the case of the Rayleigh regime. In the other fragmentation modes, there is a combination of ligament stretching and the appearance of bag breakup and stretched breakup. 
Generally speaking (not shown here), the ratio $D_{32} / D_{30}$ is between 1.5 and 2. Its values tend to approximately 1.5 for the largest values of $W e_{g}$ and smallest values of $q$ (corresponding to the lower right quarter of Fig. 3), and, on the contrary, to approximately 2 for the smallest values of $W e_{g}$ and largest values of $q$ (upper left quarter of Fig. 3). This amply justifies a more detailed analysis of the probability distributions which are only shown in an indicative way in Fig. 3.

(a)

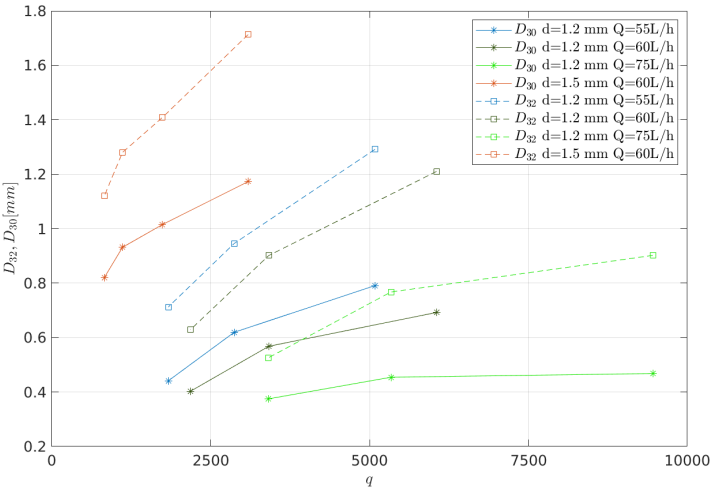

(b)

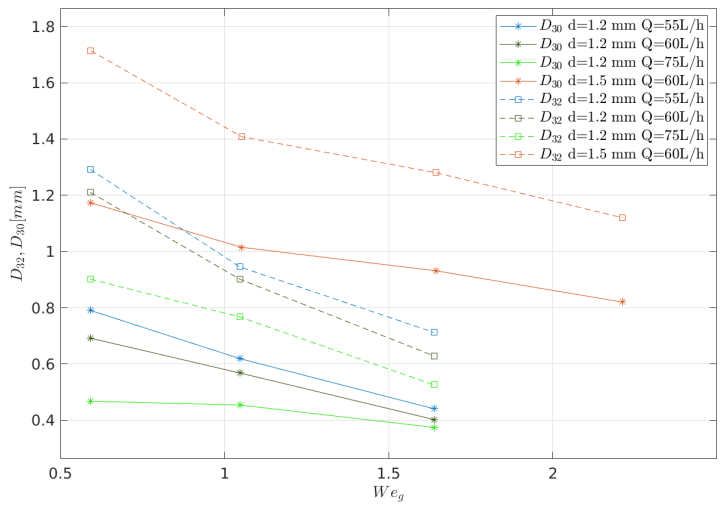

Figure 4. $D_{30}$ and $D_{32}$ as a function of (a) the momentum flux ratio and (b) the Weber aerodynamic number $W e_{g}$. Nozzles with diameters $d=1.2$ and $1.5 \mathrm{~mm}$ with flow rates of $Q=55,60$ and $75 \mathrm{~L} / \mathrm{h}$ are used.

The length of breakup was only observed in the Rayleigh regime. On one hand, the length increases with speed (see Figure 5b). The breakup radius, on the other hand, decreases with increasing rotation speed because, as shown in Figure $5 b$, the spiral is coiled more tightly with increasing angular velocity. The breakup length may be longer, but the breakup is closer to the rotation centre. This information is of great practical interest in view of the use of rotary sprinklers in confined spaces.

(a)

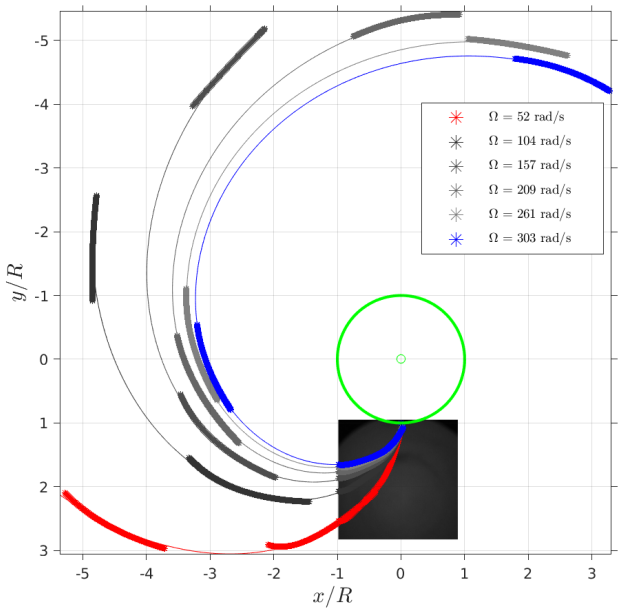

(b)

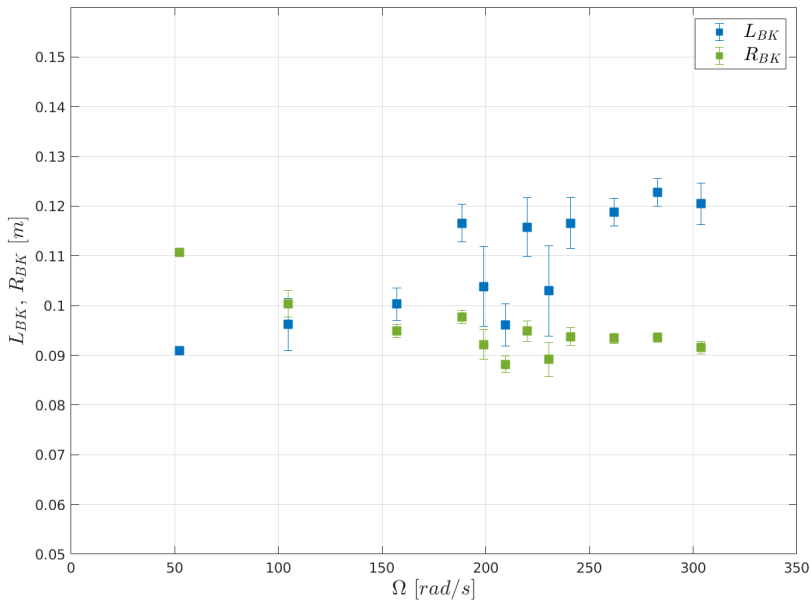

Figure 5. Overall properties of the rotating jets. (a) Pathlines for different rotation speeds. The analytical adjustment of these curves is obtained using three images: Reference image at $r / d=0$, image at breakup length, image at $r / d=125$. The conditions are in the Rayleigh regime, $\mathrm{d}=1.5 \mathrm{~mm}, \mathrm{Q}=35 \mathrm{~L} / \mathrm{h}, \mathrm{q}=[282-2369]$,

$W e_{g}=[0.07-2.21]$. (b) Breakup length $L_{B K}$ and breakup radius $R_{B K}$ for the same conditions, as a function of angular velocity $\Omega$.

\section{Conclusions and future prospects}

We have built an experimental bench that allows us to observe the different modes of rupture according to the nozzle size, flow rate and rotation speed. For each mode of rupture the experimental bench has the capacity to detect the sizes and velocities of the drops as well as the jet trajectory. In this paper we have shown the distribution of drop size for different modes and the trajectory for the Rayleigh mode. 
In the future, we will characterize more accurately the different modes of a rotating jet by including the study of particle velocity. These analyses will allow us to better characterize the mechanisms at play in the atomization of a rotating jet applied to agriculture. In particular, we will try to define the trajectory equations and measure the breakup lengths for all the breakup modes as a function of the dimensionless numbers $W e_{g}$ and $q$. The probability density of the drop size distribution is likely to be represented by a Gamma distribution for the single mode and the linear addition of two Gamma distributions for the bi-modal curves [4], [10]. We will adjust these Gamma laws for our different case studies in order to analyze the evolution with the values of $W e_{g}$ and $q$ of the parameters associated with the Gamma laws. The prediction of the size of the drops for the different fragmentation modes will allow to better control the drift and the runoff of the drops. Measurements will also be carried out for freely rotating jets, i.e. using nozzles whose rotation is not ensured by a motor, which corresponds to the situation for agricultural applications, and these results will be compared with those obtained with a motor, the situation with a motor allowing much better control of the operating conditions and the various control parameters, as well as ensuring good repeatability of the experiments. These different results will then be compared with numerical simulations under OpenFOAM carried out in a non-inertial frame of reference to simulate rotation.

\section{Acknowledgements}

This work was financed by Rolland Arroseurs sprinklers, a company specializing in irrigation equipment, as part of a joint project between the laboratories INRAE-GEAU in Montpellier and IRPHE in Marseille.

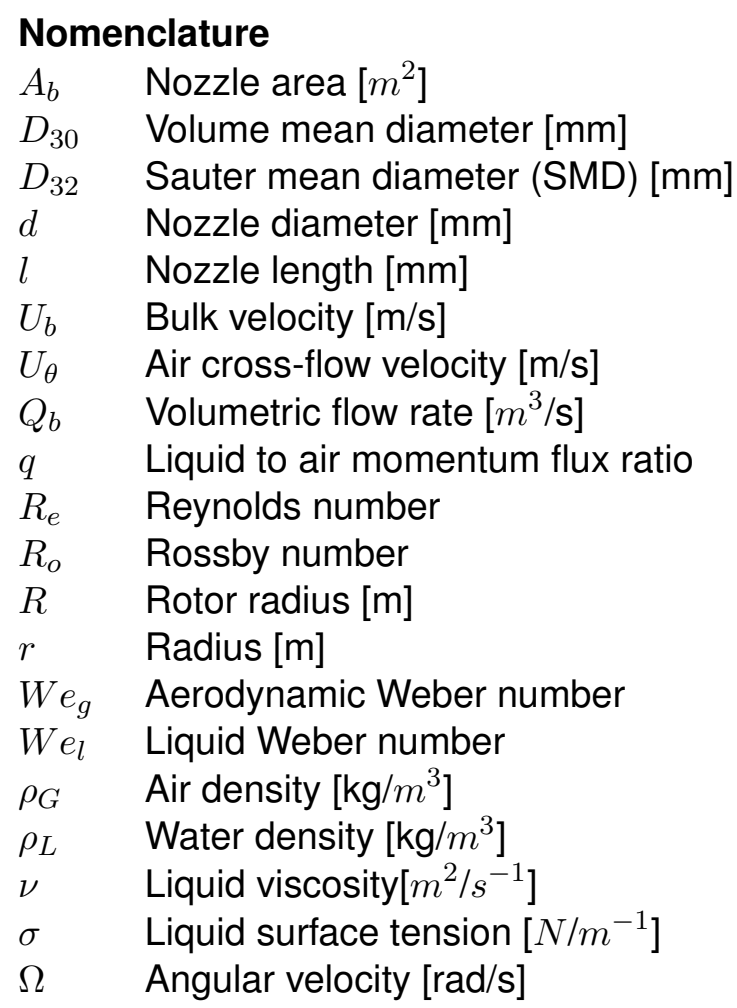




\section{References}

[1] Bayvel, L. and Orzechowski, Z., Liquid Atomization, Taylor and Francis, 1993.

[2] Felis, F., Tomas, S., Vallet, A., Amielh, M. and Anselmet, F., Int. J. of Heat and Fluid Flow, 85:108624, 2020.

[3] Felis, F., Ph D thesis, 2017.

[4] Keshavarz, B., Houze, E. C., Moore, J. R., Koener, M. R. and McKinley, G. H., Phys. Rev. Fluids, 5, 033601, 2020.

[5] Mazallon, J., Dai, Z. and Faeth, G. M., Atomization and Sprays, vol. 9, pp. 291-311, 1999.

[6] Molle, B., Tomas, S., Huet, L., Audouard, M., Olivier, Y. and Granier, J., J. Irrig. Drain. Eng., 142:04016031, 2016.

[7] Rezayat, S., Farshchi, M. and Ghorbanhoseini, M., Int. J. Multiphase Flow, vol. 111, pp. 315-338, 2018.

[8] Sallam, K. A., Aalburg, C. and Faeth, G. M., AIAA J., vol. 42, pp. 2529-2540, 2004.

[9] Stevenin, C., Vallet, A., Tomas, S., Amielh, M. and Anselmet, F., Int. J. Multiphase Flow, vol. 57, pp. 142-149, 2016.

[10] Villermaux, E. and Bossa, B., J. Fluid Mech., vol. 668, pp. 412-435, 2011. 